ARTICLE

Received 7 Oct 2015 | Accepted 1 Sep 2016 | Published 6 Oct $2016 \quad$ DOI: 10.1038/ncomms13088 OPEN

\title{
Spatiotemporal control of cell-cell reversible interactions using molecular engineering
}

Peng Shi ${ }^{1,2}$, Enguo Ju' ${ }^{1,2}$, Zhengqing Yan ${ }^{1,2}$, Nan Gao $^{1}$, Jiasi Wang ${ }^{1,2}$, Jianwen Hou $^{2}$, Yan Zhang ${ }^{3}$, Jinsong Ren ${ }^{1}$ \& Xiaogang $\mathrm{Qu}^{1}$

Manipulation of cell-cell interactions has potential applications in basic research and cell-based therapy. Herein, using a combination of metabolic glycan labelling and bioorthogonal click reaction, we engineer cell membranes with $\beta$-cyclodextrin and subsequently manipulate cell behaviours via photo-responsive host-guest recognition. With this methodology, we demonstrate reversible manipulation of cell assembly and disassembly. The method enables light-controllable reversible assembly of cell-cell adhesion, in contrast with previously reported irreversible effects, in which altered structure could not be reused. We also illustrate the utility of the method by designing a cell-based therapy. Peripheral blood mononuclear cells modified with aptamer are effectively redirected towards target cells, resulting in enhanced cell apoptosis. Our approach allows precise control of reversible cell-cell interactions and we expect that it will promote further developments of cell-based therapy.

\footnotetext{
${ }^{1}$ Laboratory of Chemical Biology and State Key Laboratory of Rare Earth Resource Utilization, Changchun Institute of Applied Chemistry, Chinese Academy of Sciences, Changchun 130022, China. ${ }^{2}$ University of Chinese Academy of Sciences, Beijing 100039, China. ${ }^{3}$ College of life science, Jilin University, Changchun 130012, China. Correspondence and requests for materials should be addressed to X.Q. (email: xqu@ciac.ac.cn or xqu@ciac.jl.cn).
} 
$\mathrm{D}$ ynamic cell-cell interactions are imperative for correct cell behaviour. The failure of cell communications can cause uncontrollable cell growth and cancer ${ }^{1,2}$. Responding cells make direct physical contact with signalling cells, 'read' their markers and give appropriate responses. For example, intercellular proximity is a critical step towards antigen presentation. Immune cells detect antigen presented on infected cell surfaces, triggering cytokine release, causing lysis or apoptosis. Therefore, spatiotemporal modulation of cell-cell interactions would benefit fundamental cell-behavioural studies, and allow unprecedented control of cell behaviour, as well as provide synthetic biological method for the design of cell-based therapy $^{3-5}$.

Apart from molecular biological techniques to genetically engineer cells ${ }^{6,7}$, in recent years, a number of non-genetic cell-surface engineering methods have been devised for the control of ligand presentation on cell surfaces ${ }^{8}$, which would greatly facilitate the ability to manipulate cellular interactions. Among them, biotin-streptavidin bridge is a general strategy, where the surfaces of two cell types are modified with a biotin-streptavidin pair, followed by the assembly of the modified cells via specific biotin-streptavidin interactions ${ }^{9,10}$. DNA has also been used as a bonding agent for cell-cell contacts $^{11,12}$. By taking advantage of metabolic labelling approach to modify cell surfaces with complementary short oligonucleotides, DNA hybridization assay has been reported to control over cell-cell interactions ${ }^{11}$. Besides, lipid-DNA aptamer conjugates have been used to modulate cell-cell adhesion on receptor-ligand binding ${ }^{12}$. Recently, methodology of liposome-to-cell fusion has been developed for delivery of bioorthogonal chemical groups to tailor cell membranes and subsequently direct the formation of multilayered cell tissues ${ }^{13-15}$. Lipid chemically self-assembled nanorings could be designed as a molecular scaffold to engineer cell surfaces and temporally control cell-cell interactions ${ }^{16}$. Thus far, the cell surfaces have been engineered to respond to temperature ${ }^{11}$, enzymolysis ${ }^{12}$, redox potential ${ }^{14}$ and chemical stimuli ${ }^{16}$, which can be used for modulating intercellular contacts. Although promising, it is still a challenge to control cell-cell interactions in time and space. Light manipulation may provide solution to this issue as it allows control over the cells from a distance with relatively high spatial and temporal precision ${ }^{17,18}$. However, the existing method relies on irreversible control, that is, once the designed structure on cell surface is altered, it cannot be regenerated for further use ${ }^{15}$. This can be overcome by engineering a photo-switchable cell surface.

Azobenzene represents a well-known class of photo-switchable compounds, the two isomers of which, the trans and cis forms, can be reversibly interconverted on photoirradiation ${ }^{19}$. Also, the molecular recognition of azobenzenes with cyclodextrins (CD) could be reversibly controlled by photoirradiation: the rodlike trans isomer forms a stable inclusion complex with $\mathrm{CD}$, while the bent cis isomer does not fit in $\mathrm{CD}^{20,21}$. The reversible photoisomerization of azobenzene has been used for dynamic control of cells and bacteria capture/release on stimuli-responsive substrates $^{22,23}$. Herein, for the first time, we extended this highly efficient photo-driven supramolecular recognition for spatiotemporal manipulation of cell-cell reversible interactions.

To realize this, tailoring cell surfaces with $\beta-C D$ is a prerequisite. Non-covalent cell-surface modification approaches based on lipid insertion and liposome-to-cell fusion have received increasing attention ${ }^{4,5,12-16}$. Although such methods are simple and efficient, using lipid anchor may suffer from the stability problem due to the dynamic nature of the phospholipid membrane. Metabolic labelling approaches have been well employed to introduce different functional groups on cell surfaces, showing powerful applications in cell surface engineering 24,25 . Unnatural monosaccharide derivatives are metabolically incorporated into cell-surface glycans, resulting in the cell surface display of bioorthogonal groups as specific chemical handles. Therefore, a series of functional components such as probes ${ }^{26-29}$, biomolecules ${ }^{30}$, and nanomaterials ${ }^{31,32}$, can be covalently attached via bioorthogonal reactions.

Herein, we take advantage of metabolic labelling approach and bio-orthogonal click reaction to tailor cell membranes with host molecules (Fig. 1a). The strategy involve feeding cells peracetylated $\mathrm{N}$-azidoacetylgalactosamine $\left(\mathrm{Ac}_{4} \mathrm{GalNAz}\right)$ to enrich cell surface glycoconjugates with the azide $\operatorname{tag}^{33}$, followed by conjugating with alkynyl and PEG-modified $\beta$-CD (alkynyl-PEG- $\beta$-CD) via a bio-orthogonal copper(I)-catalysed azide-alkyne cycloaddition (CuAAC). The $\beta$-CD modifications enable dynamic control of ligand presentation on cell membranes. If azobenzene is incorporated as switchable recognition component, as indicated in Fig. 1b, we could construct a photo-controlled reversible system. With this methodology, we firstly investigate reversible manipulation of cell assembly and disassembly with a homobifunctional cross-linking agent. Furthermore, considering that aptamers are promising recognition elements with high binding affinity to a broad range of targets, including cells ${ }^{34,35}$, we reason azobenzene-labelled aptamers (azo-aptamer) anchored on the cell surface could act as targeting ligands and induce cell-cell adhesion. It should be noted that this photo-active cell surface engineering method allows modulating intercellular contacts in space and time. More importantly, applying photo-driven host-guest recognition affords the cell-cell adhesion a novel property of reversible assembly controlled by light ${ }^{12}$, which is distinct from the irreversible effects, such as photolytic damage of original structure ${ }^{15}$. At last, we illustrate an actual use by designing a cell-based therapy. Peripheral blood mononuclear cells (PBMCs) modified with aptamer are effectively redirected towards target cells, resulting in enhanced cell apoptosis. Our approach would allow unprecedented control of cell behaviour and benefit studies of contact-dependent cell-cell communications.

\section{Results}

Cell surfaces modification with $\boldsymbol{\beta}$-CD. In our strategy, the metabolic substrate $\mathrm{Ac}_{4} \mathrm{GalNAz}$ was selected on the basis of its known incorporation into mucin-type O-linked glyco-proteins in mammalian cells via the $\mathrm{N}$-acetylgalactosamine (GalNAc) salvage pathway (Fig. 1a) ${ }^{33}$. As GalNAc was also found in certain glycolipids, chondroitin sulfate proteoglycans and as a terminal modification of some N-linked glycans, these structures might also be labelled with $\mathrm{Ac}_{4} \mathrm{GalNAz}^{36}$. MCF-7 human breast cancer cells were treated with $\mathrm{Ac}_{4} \mathrm{GalNAz}$ in culture media for three days, and the presence of azide groups on the cell surface was determined by covalent attachment of an alkynyl-based probe, FAM alkyne (Supplementary Fig. 1a). As shown in confocal fluorescence images, bright fluorescence was observed for the $\mathrm{Ac}_{4} \mathrm{GalNAz}$-treated cells when tagged with FAM alkyne by the click reaction (Supplementary Fig. 1b). In contrast, no fluorescence signal was observed for cells not exposed to $\mathrm{Ac}_{4} \mathrm{GalNAz}$ (Supplementary Fig. 1c). These results confirmed $\mathrm{Ac}_{4} \mathrm{GalNAz}$-treated cells successfully expressed cell surfaceassociated azide groups and could be chemoselectively decorated with alkynyl conjugates.

Next, to tailor cell surface with $\beta-C D$, azides installed within cell surface glycoconjugates was subjected to TBTA-assisted CuAAC with alkynyl-PEG- $\beta$-CD (Supplementary Fig. 2) to produce stable cell-surface adducts. A series of experiments were 
a

a $\quad \mathrm{Ac}_{4} \mathrm{Ga} 1 \mathrm{NAz}$

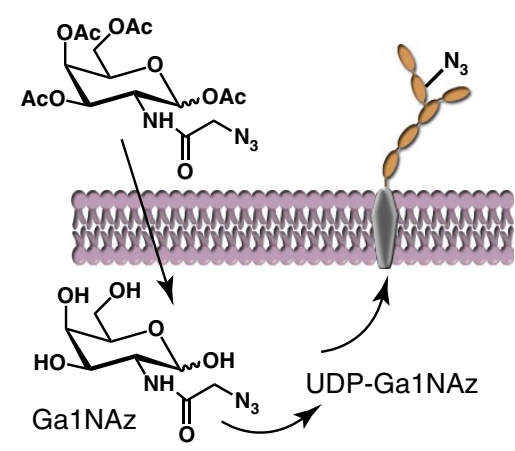

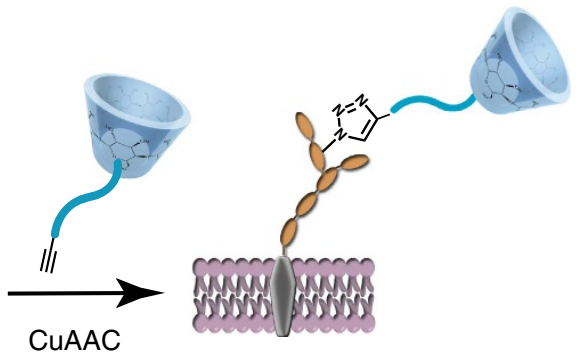

CUAAC
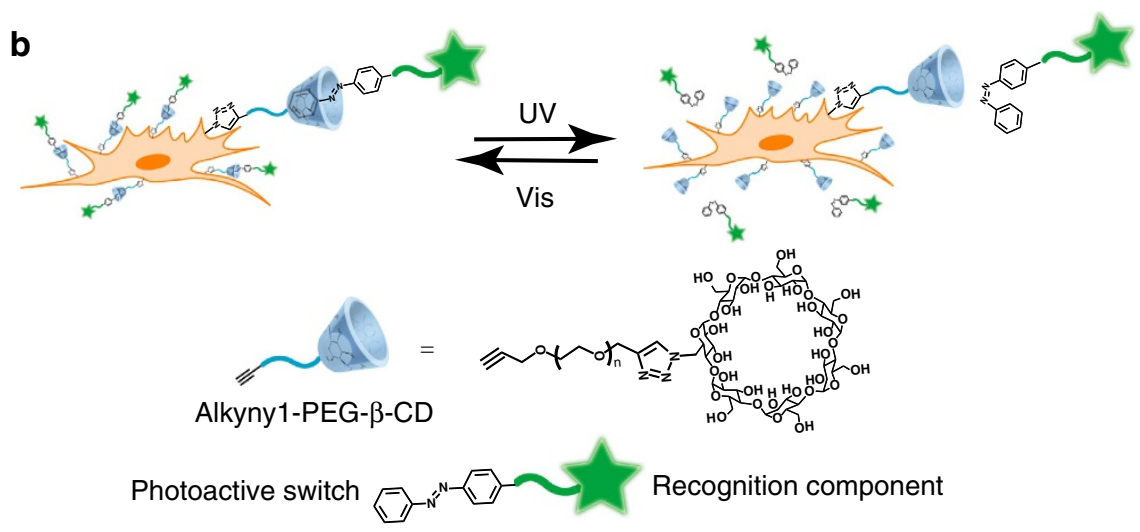

Figure 1 | Schematic illustration of engineering photo-responsive host-guest recognition on cell surfaces. (a) Metabolic labelling of mucin-type O-glycans with $\mathrm{Ac}_{4}$ GalNAz by the GalNAc salvage pathway resulted in the enrichment of the azide tag. CuAAC was used to conjugate alkynyl-PEG- $\beta$-CD. (b) Recognition component-linked azobenzenes could be built onto $\beta$-CD-conjugated cell surface through host-guest interactions to construct photo-controlled reversible systems.

performed to demonstrate the successful $\beta$-CD modification and the ability of $\beta$-CD moiety to include guests. Firstly, we modified azobenzene motif onto a FAM-labelled DNA (azo-DNA-FAM) and hypothesized that azo-DNA-FAM recognized $\beta-C D$ on the cell surface and lighted up the cell membrane (Fig. 2a). As can be seen from confocal laser scanning microscopy (CLSM) images and flow cytometry analysis (Fig. 2b,d), after incubation with azo-DNA-FAM, obvious fluorescence was observed in $\beta$-CD-modified cells. FAM signals were localized on the cell membrane. In contrast, control experiments carried out with unmodified cells showed negligible fluorescence. Moreover, competition experiments, using adamantane, were performed. Due to the higher association constant for $\beta$-CD-adamantine complex $\left(10^{4}-10^{5} \mathrm{M}^{-1}\right)$, azobenzene binding could be blocked by adamantine. As expected, no distinct azo-DNA-FAM binding was observed for $\beta$-CD-modified cell exposed to adamantine. These results confirmed the successful $\beta-C D$ modification and azobenzene binding. We thereafter quantified the number of cell-bound $\beta-C D$ through quantification of azo-DNA-FAM. $\beta$-CD modified cells were treated with azo-DNA-FAM, and subsequently analysed by flow cytometry. The mean fluorescence intensities of cells were compared with known standards (Quantum FITC MESF, Bangs Laboratories) to determine the number of azo-DNA-FAM per cell ${ }^{11}$. Control experiment of unmodified cells incubated with azo-DNA-FAM was carried out to aid in interpreting fluorescence intensity results. The results showed that $\sim 1.5 \times 10^{6}$ molecules were modified onto the cell surface (Supplementary Fig. 3). Surface $\beta-C D$ density was about 4,787 molecules per $\mu \mathrm{m}^{2}$. To further determine the half-life of $\beta$-CD on cell surface, we labelled remaining surface-associated
$\beta$-CD with azo-DNA-FAM over $72 \mathrm{~h}^{5}$. The results showed that the surface half-life was about $17.4 \mathrm{~h}$ (Supplementary Fig. 4).

According to the previous reports ${ }^{12,16,37}$, PEG linker was able to reduce nonspecific and steric interactions between the cell-surface molecules and the modified groups, thus enabling the modified molecules to protrude from the cell surface and maintain its functional conformation. To investigate the effect of PEG linker, we prepared alkynyl- $\beta$-CD (without PEG spacer) as a control (Supplementary Fig. 5). Ac $c_{4}$ GalNAz-treated MCF-7 cells were tagged with alkynyl-PEG- $\beta-C D$ or alkynyl- $\beta-C D$ by a click reaction, and subsequently coupled to azo-DNA-FAM. The cell surface labelling was explored using flow cytometry analysis. Figure $2 \mathrm{~d}$ showed that fluorescence signals in alkynyl- $\beta-C D$ modified cells were much lower than that of alkynyl-PEG- $\beta$-CD modified cells, which could be possibly attributed to the lower $\beta$-CD modification efficiency or lower azobenzene binding efficiency in alkynyl- $\beta-C D$ treated cells. In general, the above results indicate that PEG linkers play an important role in cell-surface modification. In all experiments otherwise stated, we used alkynyl-PEG- $\beta$-CD.

We next investigated the utility of $\beta$-CD on the cell surface by attaching cells to an azobenzene-patterned substrate (Supplementary Fig. 6) and releasing them again (Fig. 3a). $\beta$-CD-labelled cells were seeded to patterned substrate and cultured for $4 \mathrm{~h}$, thereafter washed with PBS and stained with green dyes. As shown in Fig. 3b, the $\beta$-CD-labelled cells could selectively attach to trans-azobenzene-patterned regions through host-guest interactions. As a control, unmodified cells did not attach to the surface (Supplementary Fig. 7). Ultraviolet light trigger was then applied to induce the isomerization of 
a

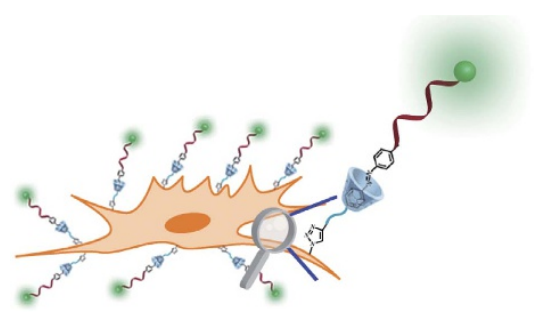

b
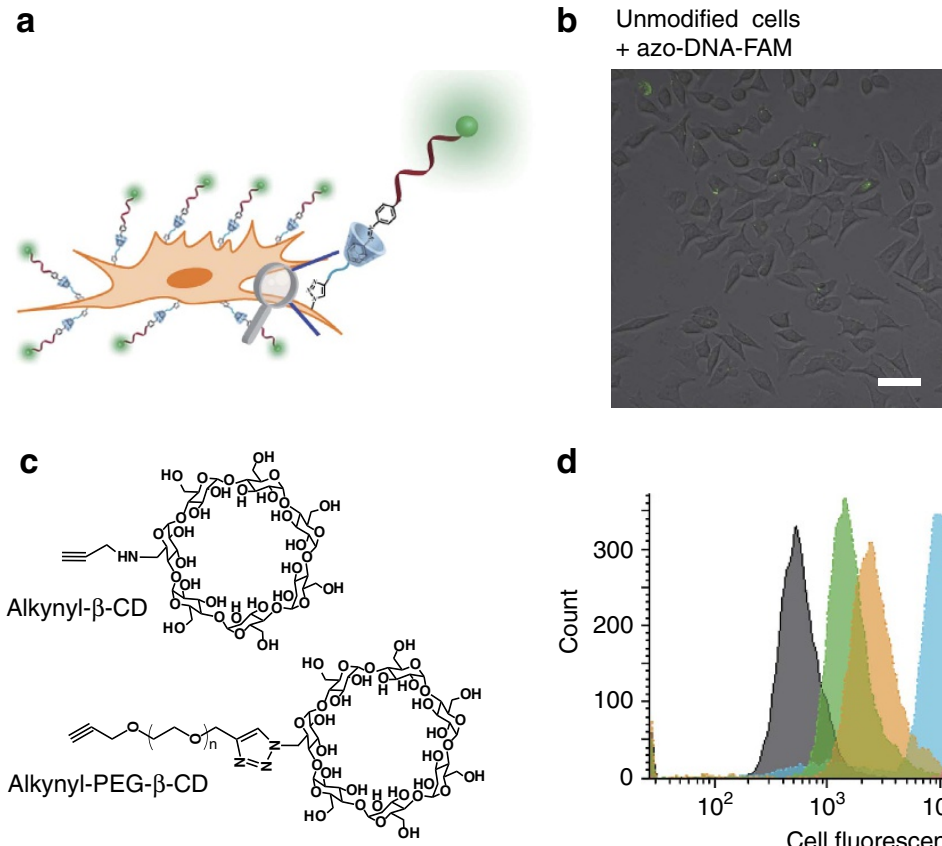

$\beta$-CD-modified cells + azo-DNA-FAM

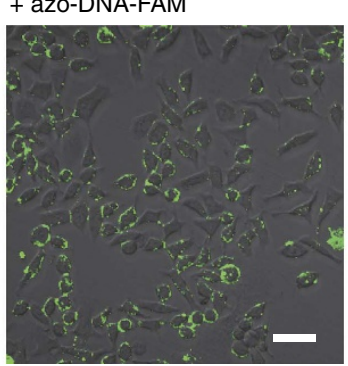

$\beta$-CD-modified cells + adamantane + azo-DNA-FAM d

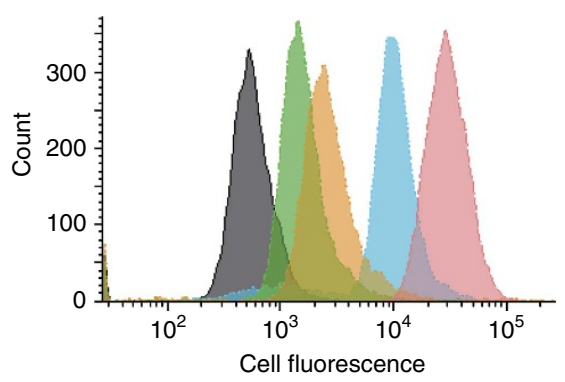

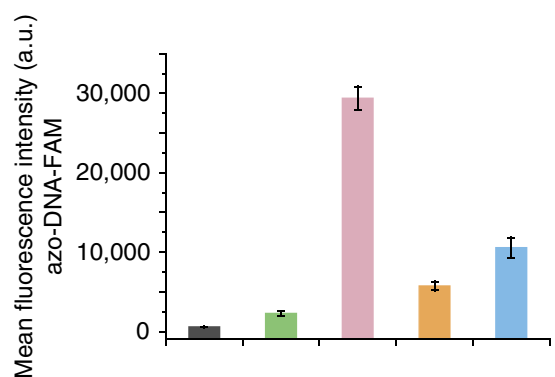

Figure 2 | Characterization of cell-surface modification by CLSM images and flow cytometry analysis. (a) Schematic illustration of cell-surface decoration with azo-DNA-FAM. (b) CLSM images of unmodified or $\beta$-CD-modified MCF-7 cells after different treatment. FAM signals were found only on the cell surface. Scale bars, $50 \mu \mathrm{m}$. (c) Chemical structure of alkynyl- $\beta-C D$ and alkynyl-PEG- $\beta-C D$. (d) Flow cytometry assay of azo-DNA-FAM binding efficiency, black: untreated MCF-7 cells, green: unmodified cells treated with azo-DNA-FAM, pink: alkynyl-PEG- $\beta$-CD modified cells treated with azo-DNA-FAM, orange: alkynyl-PEG- $\beta$-CD modified cells treated with adamantine followed by azo-DNA-FAM, blue: alkynyl- $\beta$-CD modified cells treated with azo-DNA-FAM. Data were presented as mean \pm s.d $(n=3)$.

azobenzene, from trans-isomer to the cis-isomer. As a result, cells would detach from the substrate because of the mismatch between the host and guest. As expected, just $\sim 14 \%$ of the adherent cells were released without ultraviolet irradiation. However, after exposure to ultraviolet light and washing with PBS, $\sim 84 \%$ of the original adherent cells were detached from the substrate. (Fig. 3c,d). Such a strategy enables a synthetic approach to spatio-temporally manipulate cells in $2 \mathrm{D}$ and may be extended for tissue engineering applications. Besides, we prepared azobenzene and PEG-modified fluorescent silica nanoparticle (azo-PEG-SiNP, Supplementary Fig. 8) and tested the reversible binding of azo-PEG-SiNP to the $\beta$-CD-modified cell surfaces (Supplementary Fig. 9a). The $\beta$-CD-labelled cells were first fixed, then incubated with azo-PEG-SiNP for $20 \mathrm{~min}$, washed three times by PBS and imaged on confocal fluorescence microscope. The results demonstrated modified cell surfaces were lighted up by azo-PEG-SiNP (Supplementary Fig. 9b). Scanning electron microscopy (SEM) also confirmed attachment of nanoparticles on the cell surfaces (Supplementary Fig. 9c). In contrast, azo-PEG-SiNP showed minimal binding to non-labelled cells under identical conditions as illustrated by negligible fluorescence (Supplementary Fig. 10). Importantly, noncovalent nature of host-guest interactions allowed detachment of the imaging agent. On ultraviolet irradiation, azo-PEG-SiNP was released from the cell surfaces and negligible labelling was observed (Supplementary Fig. 10d). The photocontrolled attachment and detachment of azo-PEG-SiNP also confirmed the successful presentation of $\beta-C D$ on cell surface.

Reversible manipulation of cell assembly and disassembly. The presentation of $\beta-\mathrm{CD}$ on the cell membrane enabled controlled display of functional components through host-guest interactions. We extended this methodology to demonstrate dynamic control over cell-cell interactions. Before the experiments, we checked the cell viability before and after bioorthogonal click reaction. $\mathrm{Ac}_{4} \mathrm{GalNAz}$ treatment had little effect on cell viability at a nontoxic concentration of $50 \times 10^{-6} \mathrm{M}$. The subsequent modification with alkynyl-PEG- $\beta$-CD resulted in a slight decrease of cell viability. Besides, the viability of the cells did not change obviously when treated with 10 min ultraviolet irradiation (365 nm, 15 W) (Supplementary Fig. 11). Next, we used a homobifunctional guest molecule (azo-PEG-azo) which carried two identical supramolecular binding sites: azobenzene groups (Supplementary Fig. 12). By means of isothermal titration calorimetry (ITC), the interaction of the guests with $\beta$-CD was examined. It was found that azo-PEG-azo formed complexes with $\beta$-CD in a 1:2 ratio, with association constant of $8.62 \times 10^{3} \mathrm{M}^{-1}$ (Supplementary Fig. 13). The photoisomerization of azo-PEG-azo was also investigated (Supplementary Fig. 14). We demonstrated this homo-bifunctional cross-linking agent served as 'reversible cell glues ${ }^{38}$, could induce adhesion and aggregation of $\beta$-CD-modified cells. Cell assembly and disassembly could be reversibly manipulated with light irradiation (Fig. 4a). The $\beta$-CD modified MCF-7 cells were divided into two subsets. For tracking purposes, the two subsets of cells were labelled with green or red dyes respectively, so that the cell clustering could be easily observed. When mixed together at $1: 1$ ratio in the absence of azo-PEG-azo, cells were well separated and no aggregates were observed. However, addition of azo-PEG-azo caused apparent cell aggregation (Fig. .4b; Supplementary Fig. 15a). Both green- and red-stained cells were present in the same cell cluster. To further confirm that the cell assembly was attributed to supramolecular interactions, a series of control experiments were added. As shown in Supplementary Fig. 15, cell binding did not occur without the proper complementary host-guest pair. Moreover, competition experiments, using adamantane, were also performed. $\beta$-CD-modified cells were firstly exposed to 

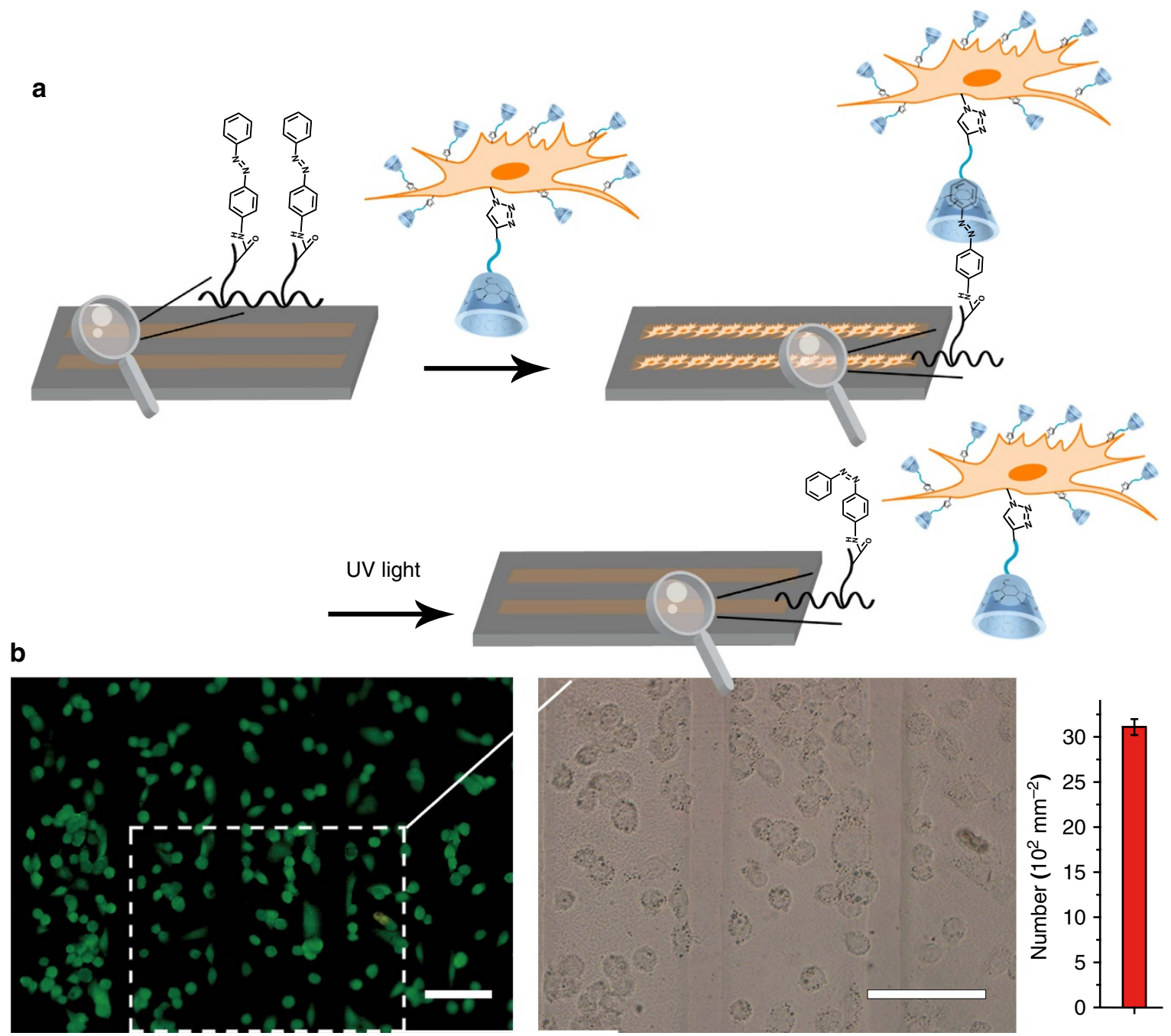

c

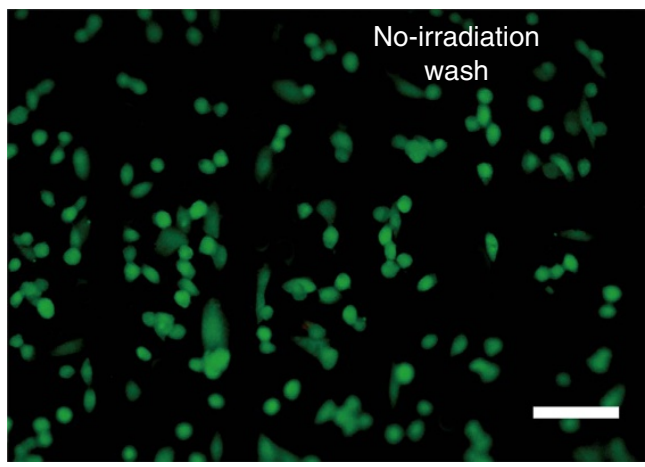

d

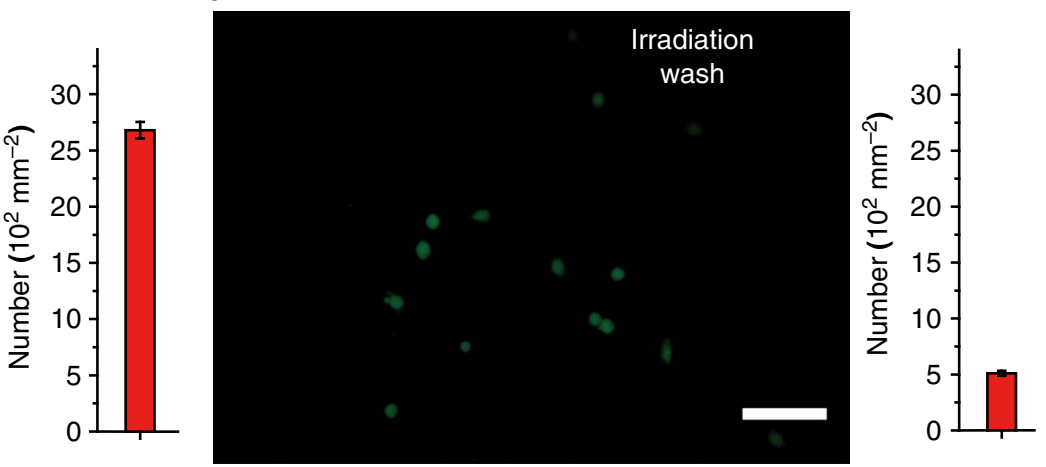

Figure 3 | Fluorescence images and quantitative analysis of controlled cell adhesion and release. (a) Outline of attaching $\beta$-CD-labelled cells to an azobenzene-patterned substrate and releasing them again. (b) Fluorescence image (left) and bright-field image at higher magnification (right) indicated $\beta$-CD-labelled cells could selectively attach to trans-azobenzene-patterned regions. Cells on the substrate were treated with (d) or without (c) ultraviolet irradiation $(365 \mathrm{~nm}, 15 \mathrm{~W}, 10 \mathrm{~min}$ ) for the selective release. Scale bars, $50 \mu \mathrm{m}$. Data were presented as mean \pm s.d. from three independent experiments.

adamantine, followed by azo-PEG-azo. As a result, no cell aggregation was observed, which indicated azobenzene binding could be blocked by adamantine. All above results confirmed that the cell binding was attributed to supramolecular interactions.

Due to the remarkable difference in the binding affinity between $\beta-C D$ and trans-azobenzene (high) and $\beta-C D$ and cis-azobenzene (low), photoinduced dispersion and re-aggregation of the cells could be achieved by ultraviolet irradiation, followed by Vis irradiation (Fig. 4b). This novel property of lightcontrolled reversible assembly was attributed to the noncovalent nature of host-guest interactions. To give more details, real-time monitoring of light-induced cell disassembly was performed. The cell aggregates were irradiated with ultraviolet light $(365 \mathrm{~nm}$, $15 \mathrm{~W}$ ), and the image was recorded every $60 \mathrm{~s}$. As shown in Fig. 5a, looseness and disassembly of the cell cluster were gradually observed with time increasing. When time came to 

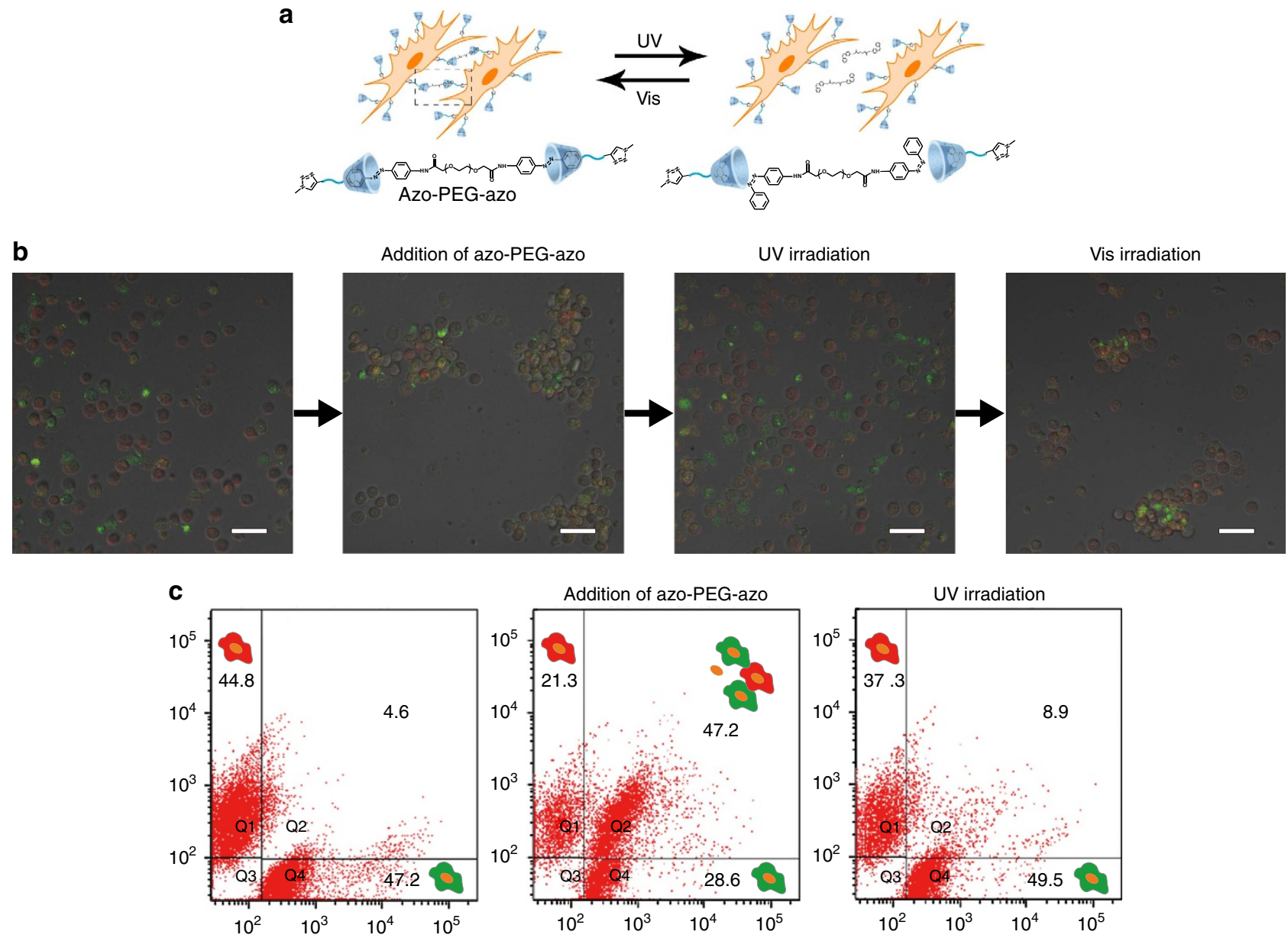

Figure 4 | Reversible manipulation of cell assembly and disassembly. (a) Schematic illustration describing reversible cell-cell contacts. A homobifunctional guest molecule served as 'reversible cell glues' could induce assembly and disassembly of $\beta$-CD-modified cells through light-manipulation. The rod-like trans-isomer formed a stable inclusion complex with $\beta$-CD, while the bent cis-isomer did not fit in $\beta$-CD. (b) CLSM images and (c) flow cytometry analysis of $\beta$-CD-modified cells with different treatment. Scale bars, $50 \mu \mathrm{m}$.

$540 \mathrm{~s}$, the cell disassembly was completed. The control experiment without ultraviolet irradiation was also carried out at the same condition (Fig. 5b). Although the morphology of the cell cluster was changed, no obvious cell disassembly was observed. The time scanning images clearly demonstrated the process of light-induced cell disassembly. At last, quantitative analysis of the photo-controlled manipulation of cell assembly was added using flow cytometry (Fig. 4c). In the absence of azo-PEG-azo, the flow cytometry data did not show any aggregates in the upper right quadrant of the scatters, which meant most cells were separated. As expected, a new data cluster representing cellular aggregates appeared in the scatter when addition of $20 \mu \mathrm{M}$ azo-PEG-azo. The cluster represented $47.2 \%$ of cells formed aggregates. On ultraviolet irradiation, the data cluster disappeared, with $8.9 \%$ cell aggregates remained, which meant photo-induced dispersion of the cells was achieved. We have also quantified the percentage of azo-PEG-azo bound on the same cell surface versus trans-cellular. The results showed about $71 \%$ of azo-PEG-azo used for trans-cellular binding (Supplementary Fig. 16).

Heterotypic cell adhesion and cell-based therapy. Furthermore, we aimed at endowing cell-cell contacts with targeting property. Considering the high binding affinity of aptamer to their targets, we prepared azo-aptamer as a photo-switchable recognition component and anchored it on the cell surface as binding agents to dynamic control over cell-cell interactions (Fig. 6a). In this experiment, aptamer MUC 1, which targets mucin 1 protein expressed on epithelial cancer cells (MCF-7; MUC 1+), was used for testing ${ }^{35}$. The aptamer exhibited high affinity towards their target cells by mimicking native cell-surface ligand-receptor interactions. We expected that Hela cells modified with MUC 1 aptamer would recognize their target cells MCF-7 and form a cell-aptamer-cell assembly. For easily distinguishing, Hela cells and MCF-7 cells were stained with green and red dyes respectively. As can be seen from Fig. 6b,c, after washing, MCF-7 cells could not adhere to unmodified Hela cells. By contrast, heterotypic cell adhesions were successfully observed between MCF-7 cells and MUC 1 aptamer-presenting Hela cells. Scanning electron microscopic (SEM) images gave detailed three-dimensional morphology of cellular interactions. The cellular morphologies were well maintained, and direct contact at cell-cell interfaces was clearly observed (Fig. 6f). As mentioned above, an advantage of introducing photo-responsive host-guest recognition is the potential for controlled photoirradiation to reverse the linkage. ultraviolet irradiation induces the photoisomerization of trans-isomer to cis-isomer. Since cis-azobenzenes cannot form inclusion complexes with $\beta$-CD, azo-aptamers are no longer displayed on the cell surface, resulting in disassociation of cell-cell contacts. As shown in Fig. 6d, on ultraviolet irradiation, MCF-7 cells were released from the surface 
a + Irradiation

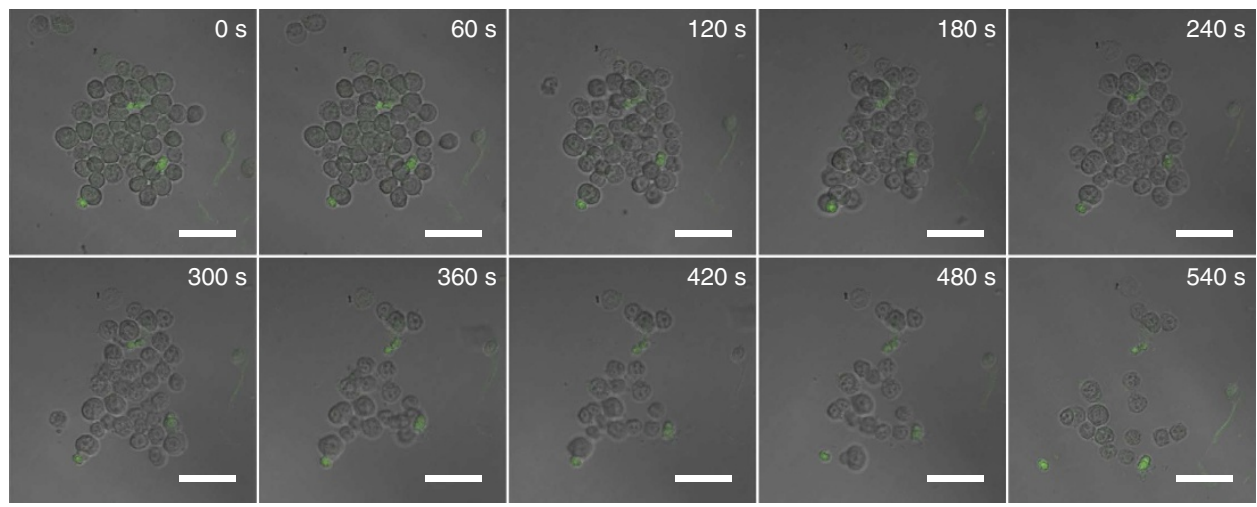

b - Irradiation

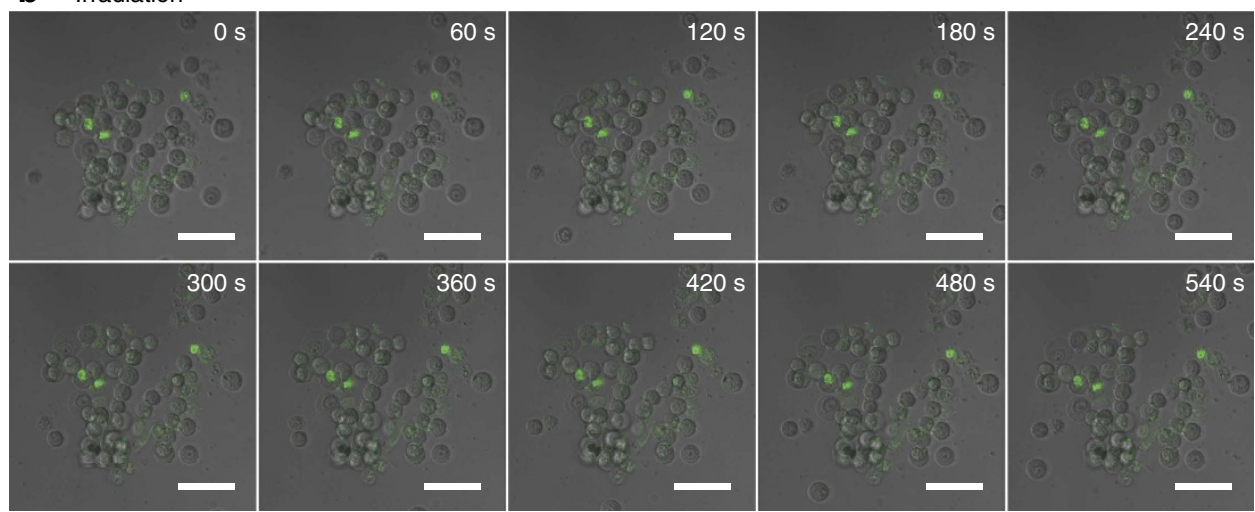

Figure 5 | Real-time monitoring of light-induced cell disassembly. Real-time CLSM images of cell cluster (a) treated with or (b) without ultraviolet irradiation $\left(365 \mathrm{~nm}, 15 \mathrm{~W}\right.$ ). Flow at $1 \mathrm{ml} \mathrm{min}^{-1}$ was applied for the process. Scale bars, $50 \mu \mathrm{m}$.

of Hela cells. We further demonstrated that $\beta$-CD-labeled Hela cells could be once again modified with azo-aptamer and used for cell-cell adhesion (Fig. 6e). Although aptamer has also been used as a binding agent for cell-cell contacts ${ }^{12}$, our attractive method exhibited obvious advantage over the previous reported irreversible effects, in which the altered structure could not be reused. At last, we illustrated an actual use by applying our method toward directing PBMCs to induce target cell apoptosis. Human PBMCs were obtained through density gradient centrifugation and modified with aptamer MUC 1 using the above labelling strategy. The resulted aptamer-modified PBMCs or unmodified PBMCs were co-incubated with MCF-7 cells, respectively, and shaken at $37^{\circ} \mathrm{C}$. The cell-cell contacts were viewed using optical microscope. As can been seen from Fig. 6g,h, compared with unmodified PBMCs, aptamer-modified PBMCs could effectively adhere to the target MCF-7 cells and form $\mathrm{T}$ cells-cancer cells assembly. We also estimated the efficiency of PBMCs-induced cell apoptosis by lactate dehydrogenase release assay $^{16}$, which indicated aptamer-modified PBMCs showed enhanced cytotoxicity to MCF-7 cells (Supplementary Fig. 17).

\section{Discussion}

We engineered cell membranes with $\beta$-CD for dynamic control of cell-cell interactions using a combination of metabolic glycan labelling and bio-orthogonal click reaction. Furthermore, the aggregation and dispersion of cells were mediated by a fully reversible photo-responsive molecular recognition process. Three important features are associated with our photo-switchable cell surface engineering system: (1) this method allows modulating intercellular contacts in space and time; (2) cell-cell adhesion can be reversible controlled by light; (3) due to the presence of $\beta$-CD, we envision that a series of stimuli-responsive host-guest recognition can be introduced to meet multi-level demand. Our design opens a new avenue to control contact-dependent cell-cell reversible interactions and will promote further studies on cell communications.

\section{Methods}

Cell labelling experiments. $\mathrm{N}$-azidoacetylgalactosamine ( $\left.\mathrm{Ac}_{4} \mathrm{GalNAz}\right)$ was purchased from Invitrogen (Eugene, OR, USA). FAM alkyne and Copper(II)-TBTA complex (10 mM in 55\% aq. dimethylsulfoxide) were purchased from Lumiprobe. MCF-7 cells/Hela cells were cultured using the general DMEM medium containing $50 \mu \mathrm{M} \mathrm{Ac}_{4} \mathrm{GalNAz}$ for three days to enrich the azido groups in $\mathrm{O}$-linked glycoproteins. The $\mathrm{Ac}_{4} \mathrm{GalNAz}$-labelled cells were washed three times with IX PBS, then incubated with PBS containing $50 \mu \mathrm{M}$ Copper(II)-TBTA complex, $2 \mathrm{mM}$ sodium ascorbate, $25 \mu \mathrm{M}$ FAM alkyne (or $50 \mu \mathrm{M}$ alkynyl-PEG- $\beta-C D$ ) in the dark at $\mathrm{rt}$ for $10 \mathrm{~min}$, followed by three washes before being used for the following experiments. The $\beta-\mathrm{CD}$ modified cells were incubated with azo-DNA-FAM $(25 \mu \mathrm{M})$ for $60 \mathrm{~min}$, followed by three washes before fluorescence imaging and flow cytometry analysis. Fluorescence imaging experiments were performed on a Zeiss LSM700 confocal laser scanning microscope. Flow cytometry analysis was performed on BD FACS Aria. For experiment of attaching $\beta$-CD-labelled cells to an azobenzene-patterned substrate, the $\beta$-CD-labelled cells were detached from 6-well plates by incubation with $1 \mathrm{mM}$ EDTA-PBS for $10 \mathrm{~min}$ at $37^{\circ} \mathrm{C}$. Then cells were incubated with the modified substrates for $4 \mathrm{~h}$. The unbound cells were gently removed by rinsing with buffer for $1 \mathrm{~min}$. Cells were stained by AM fluorescent dye for $10 \mathrm{~min}$ and viewed using an Olympus BX-51 optical system microscope (Tokyo, Japan). For ultraviolet triggered release, cell-attached substrate were irradiated with ultraviolet light $(365 \mathrm{~nm}, 15 \mathrm{~W})$ for $10 \mathrm{~min}^{39}$. After that, the substrate was gently washing with PBS for $15 \mathrm{~s}$ to remove the released cells. Then it was measured with fluorescence images to monitor the cell release.

Cell assembly and disassembly. For investigating reversible manipulation of cell assembly and disassembly with the homobifunctional guest molecule 
a Azobenzene-Aptamer
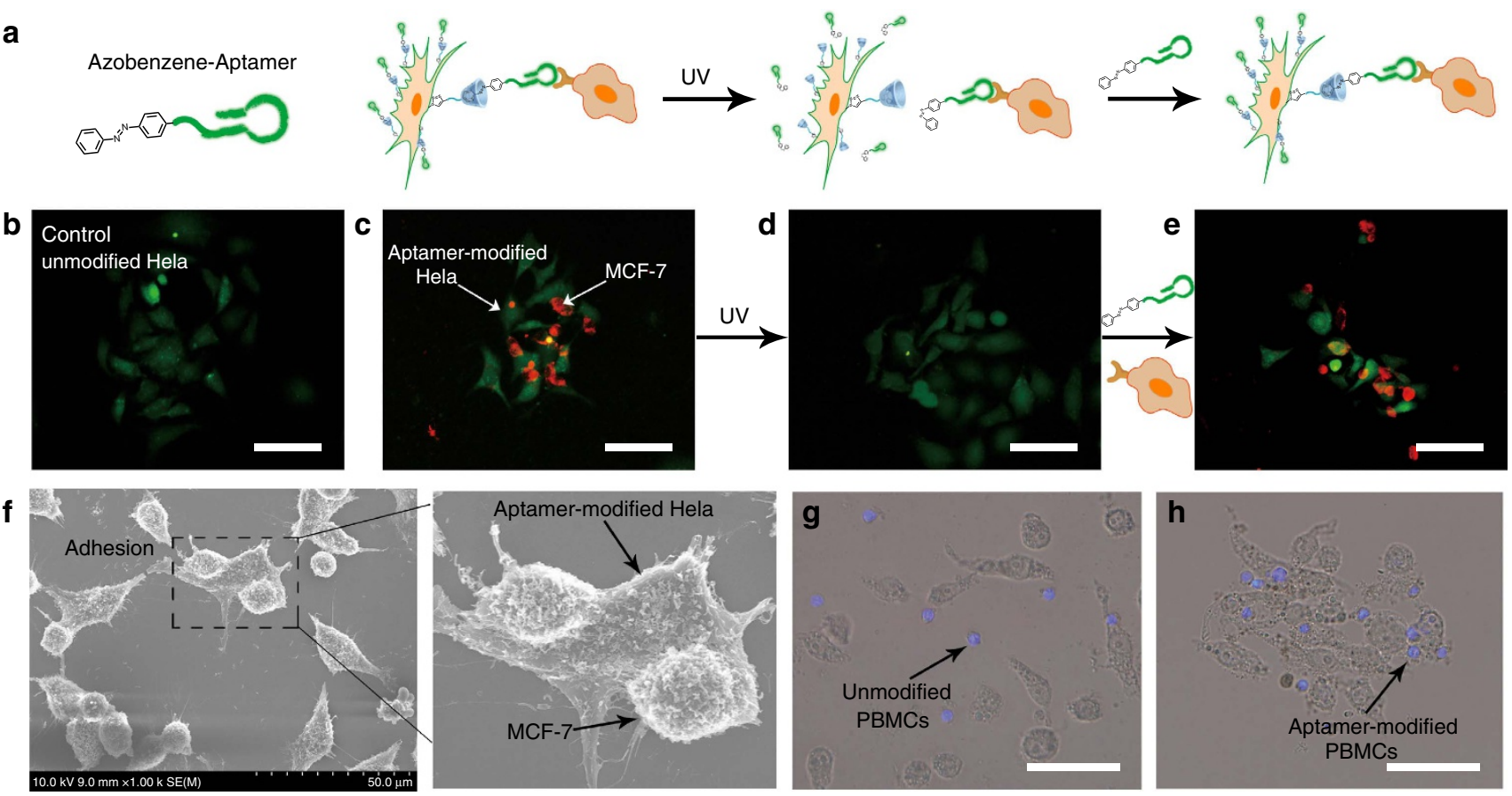

Figure 6 | Fluorescence images and SEM images of heterotypic cell adhesion. (a) Azobenzene-labelled-aptamers (azo-aptamer) anchored on the cell surface could act as targeting ligands that specifically recognized their target cells and induced cell-cell adhesion. MUC 1 aptamer-modified Hela cell (green) could recognize the target cells MCF-7 (red) and form a cell-aptamer-cell assembly. (b,c) Fluorescence images of heterotypic cell adhesions between unmodified Hela (b), modified Hela (c) and MCF-7. (d) After ultraviolet irradiation ( $365 \mathrm{~nm}, 15 \mathrm{~W}, 10$ min) and washing with PBS, MCF-7 cells were released from the surface of Hela cells. (e) Hela cells could be once again modified with azo-aptamer and used for cell-cell adhesion. (f) SEM images of cellular interactions. Cell-cell adhesion was clearly observed. $(\mathbf{g}, \mathbf{h})$ Microscope images of heterotypic cell adhesions between unmodified PBMCs (g), modified PBMCs (h) and MCF-7. Scale bars, $50 \mu \mathrm{m}$.

(azo-PEG-azo), the $\beta$-CD-labelled cells were detached from 6-well plates by incubation with $1 \mathrm{mM}$ EDTA-PBS for $10 \mathrm{~min}$ at $37^{\circ} \mathrm{C}$. Green-stained and red-stained $\beta$-CD-modified MCF-7 were mixed together at a 1:1 ratio in the presence of different concentrations of azo-PEG-azo, and shaken at $300 \mathrm{rpm}$ for $60 \mathrm{~min}$ at $25^{\circ} \mathrm{C}$. Aliquots were analysed by optical microscope. For ultraviolet triggered disassembly, cell aggregates were irradiated with ultraviolet light $(365 \mathrm{~nm}, 15 \mathrm{~W})$ for $10 \mathrm{~min}$ and shaken in the dark at $300 \mathrm{rpm}$ for $30 \mathrm{~min}$ at $25^{\circ} \mathrm{C}$. Aliquots were analysed by optical microscope. Flow cytometric analysis was performed on BD FACS Aria.

Heterotypic cell adhesions. The $\beta$-CD-modified Hela cells were incubated with $20 \mu \mathrm{M}$ azobenzene labelled MUC 1 aptamer for $20 \mathrm{~min}$. After washing, MCF-7 cells were added for heterotypic cell adhesions. For easily distinguishing, Hela cells and MCF-7 cells were stained with green (fluorescein) and red (lissamine rhodamine B) dyes respectively. For ultraviolet triggered release, the sample was irradiated with ultraviolet light $(365 \mathrm{~nm}, 15 \mathrm{~W})$ for $10 \mathrm{~min}$. After that, the substrate was gently washing with PBS for $15 \mathrm{~s}$ to remove the released cells.

Human peripheral blood mononuclear cells (PBMCs) were obtained through density gradient centrifugation and cultured with RPMI-1640 media, followed by modified with aptamer MUC 1 through the above labelling strategy. Briefly, PBMCs were cultured with RPMI-1640 containing $50 \mu \mathrm{M} \mathrm{Ac}_{4} \mathrm{GalNAz}$ for three days to enrich the azido groups. The azido-labelled cells were washed three times with PBS, then incubated with PBS containing $50 \mu \mathrm{M}$ Copper(II)-TBTA complex, $2 \mathrm{mM}$ sodium ascorbate, $50 \mu \mathrm{M}$ alkynyl-PEG- $\beta$-CD in the dark at $\mathrm{rt}$ for $10 \mathrm{~min}$, followed by three washes. Then the $\beta$-CD-modified PBMCs were incubated with $20 \mu \mathrm{M}$ azobenzene labelled MUC 1 aptamer for $20 \mathrm{~min}$. After washing, the resulted aptamer-modified PBMCs (or unmodified PBMCs) were added to each well of the 6-well plate containing MCF-7 cells. MCF-7 cells and PBMCs were then allowed to incubate together at $37^{\circ} \mathrm{C}$ for one hour (shaken at $300 \mathrm{rpm}$ ) and further longer. The cell-cell contacts and resulting cell lysis were viewed using an Olympus BX-51 optical system microscope (Tokyo, Japan). Measurement of cytotoxicity of MCF-7 cells on incubation with aptamer-modified or unmodified PBMCs was done using a nonradioactive cytotoxicity assay (CytoTox $96^{\mathbb{R}}$ Non-Radioactive Cytotoxicity Assay) ${ }^{16}$ that measured the amount of lactate dehydrogenase (LDH) enzyme following cell lysis.

SEM measurement. To gain more insight on the details, SEM characterization was included in our study. The specimens were fixed with $4 \%$ glutaraldehyde for $3 \mathrm{~h}$. Next, the specimens were washed with sterile water for three times and then were dehydrated by addition of ethanol in a graded series ( $15 \%$ for $15 \mathrm{~min}, 30 \%$ for $15 \mathrm{~min}, 50 \%$ for $15 \mathrm{~min}, 70 \%$ for $15 \mathrm{~min}, 80 \%$ for $15 \mathrm{~min}, 90 \%$ for $15 \mathrm{~min}$ and $100 \%$ for $15 \mathrm{~min}$ ) and then treated with tert-butanol. After drying under vacuum, the specimens were coated with platinum and examination on SEM.

Data availability. The authors declare that the data supporting the findings of this study are available from the corresponding author on request.

\section{References}

1. Friedl, P. \& Zallen, J. A. Dynamics of cell-cell and cell-matrix interactions in morphogenesis, regeneration and cancer. Curr. Opin. Cell Biol. 22, 557-559 (2010).

2. Nelson, C. M. \& Bissell, M. J. Of extracellular matrix, scaffolds, and signalling: tissue architecture regulates development, homeostasis, and cancer. Annu. Rev Cell Dev. Biol. 22, 287-309 (2006).

3. Sackstein, R. et al. Ex vivo glycan engineering of $\mathrm{CD} 44$ programs human multipotent mesenchymal stromal cell trafficking to bone. Nat. Med. 14, 181-187 (2008).

4. Jeong, J. H. et al. Leukocyte-mimicking stem cell delivery via in situ coating of cells with a bioactive hyperbranched polyglycerol. J. Am. Chem. Soc. 135, 8770-8773 (2013).

5. Hudak, J. E., Canham, S. M. \& Bertozzi, C. R. Glycocalyx engineering reveals a Siglec-based mechanism for NK cell immunoevasion. Nat. Chem. Biol. 10, 69-75 (2014)

6. Jena, B., Dotti, G. \& Cooper, L. J. N. Redirecting T-cell specificity by introducing a tumor-specific chimeric antigen receptor. Blood 116, 1035-1044 (2010).

7. Kruschinski, A. et al. Engineering antigen-specific primary human NK cells against HER-2 positive carcinomas. Proc. Natl Acad. Sci. USA 105, 17481-17486 (2008).

8. Stephan, M. T. \& Irvine, D. J. Enhancing cell therapies from the outside in: cell surface engineering using synthetic nanomaterials. Nano Today 6, 309-325 (2011).

9. Sarkar, D. et al. Engineered cell homing. Blood 118, e184-e191 (2011).

10. Wang, B. et al. Multicellular assembly and light-regulation of cell-cell communication by conjugated polymer materials. Adv. Mater. 26, 2371-2375 (2014). 
11. Gartner, Z. J. \& Bertozzi, C. R. Programmed assembly of 3-dimensional microtissues with defined cellular connectivity. Proc. Natl Acad. Sci. USA 106, 4606-4610 (2009).

12. Xiong, X. et al. DNA aptamer-mediated cell targeting. Angew. Chem. Int. Ed. 52, 1472-1476 (2013).

13. Dutta, D., Pulsipher, A., Luo, W. \& Yousaf, M. N. Synthetic chemoselective rewiring of cell surfaces: generation of three-dimensional tissue structures. J. Am. Chem. Soc. 133, 8704-8713 (2011).

14. Pulsipher, A., Dutta, D., Luo, W. \& Yousaf, M. N. Cell-surface engineering by a conjugation-and-release approach based on the formation and cleavage of oxime linkages upon mild electrochemical oxidation and reduction. Angew. Chem. Int. Ed. 53, 9487-9492 (2014).

15. Luo, W., Pulsipher, A., Dutta, D., Lamb, B. M. \& Yousaf, M. N. Remote control of tissue interactions via engineered photo-switchable cell surfaces. Sci. Rep. 4, 6313-6320 (2014)

16. Gabrielse, K. et al. Reversible re-programing of cell-cell interactions. Angew. Chem. Int. Ed. 53, 5112-5116 (2014).

17. Li, W., Wang, J., Ren, J. \& Qu, X. Near-infrared- and pH-responsive system for reversible cell adhesion using graphene/gold nanorods functionalized with i-Motif DNA. Angew. Chem. Int. Ed. 52, 6726-6730 (2013).

18. Li, W., Wang, J., Ren, J. \& Qu, X. Near-infrared upconversion controls photocaged cell adhesion. J. Am. Chem. Soc. 136, 2248-2251 (2014).

19. Mayer, G. \& Heckel, A. Biologically active molecules with a 'Light Switch'. Angew. Chem. Int. Ed. 45, 4900-4921 (2006).

20. Samanta, A., Stuart, M. C. A. \& Ravoo, B. J. Photoresponsive capture and release of lectins in multilamellar complexes. J. Am. Chem. Soc. 134, 19909-19914 (2012)

21. Nalluri, S. K. M., Voskuhl, J., Bultema, J. B., Boekema, E. J. \& Ravoo, B. J. Lightresponsive capture and release of DNA in a ternary supramolecular complex. Angew. Chem. Int. Ed. 50, 9747-9751 (2011).

22. Auernheimer, J., Dahmen, C., Hersel, U., Bausch, A. \& Kessler, H. Photoswitched cell adhesion on surfaces with RGD peptides. J. Am. Chem. Soc. 127, 16107-16110 (2005).

23. Weber, T. et al. Switching of bacterial adhesion to a glycosylated surface by reversible reorientation of the carbohydrate ligand. Angew. Chem. Int. Ed. 53, 14583-14586 (2014)

24. Mahal, L. K., Yarema, K. J. \& Bertozzi, C. R. Engineering chemical reactivity on cell surfaces through oligosaccharide biosynthesis. Science 276, 1125-1128 (1997).

25. Saxon, E. \& Bertozzi, C. R. Cell surface engineering by a modified staudinger reaction. Science 287, 2007-2010 (2000).

26. Haga, Y. et al. Visualizing specific protein glycoforms by transmembrane fluorescence resonance energy transfer. Nat. Commun. 3, 907 (2012).

27. Rong, J. et al. Glycan imaging in intact rat hearts and glycoproteomic analysis reveal the upregulation of sialylation during cardiac hypertrophy. J. Am. Chem. Soc. 136, 17468-17476 (2014)

28. Niederwieser, A. et al. Two-color glycan labeling of live cells by a combination of diels-alder and click chemistry. Angew. Chem. Int. Ed. 52, 4265-4268 (2013).

29. Lin, L. et al. A bioorthogonal raman reporter strategy for SERS detection of glycans on live cells. Angew. Chem. Int. Ed. 52, 7266-7271 (2013).

30. Chandra, R. A., Douglas, E. S., Mathies, R. A., Bertozzi, C. R. \& Francis, M. B. Programmable cell adhesion encoded by DNA hybridization. Angew. Chem. Int. Ed. 45, 896-901 (2006).

31. Wu, C. et al. Ultrabright and bioorthogonal labeling of cellular targets using semiconducting polymer dots and click chemistry. Angew. Chem. Int. Ed. 49, 9436-9440 (2010)
32. Koo, H. et al. Bioorthogonal Copper-Free Click Chemistry In Vivo for Tumor-Targeted Delivery of Nanoparticles. Angew. Chem. Int. Ed. 51, 11836-11840 (2012).

33. Hang, H. C., Yu, C., Kato, D. L. \& Bertozzi, C. R. A metabolic labeling approach toward proteomic analysis of mucin-type O-linked glycosylation. Proc. Natl Acad. Sci. USA 100, 14846-14851 (2003).

34. You, M., Zhu, G., Chen, T., Donovan, M. J. \& Tan, W. Programmable and multiparameter DNA-based logic platform for cancer recognition and targeted therapy. J. Am. Chem. Soc. 137, 667-674 (2015).

35. Hu, R. et al. DNA nanoflowers for multiplexed cellular imaging and traceable targeted drug delivery. Angew. Chem. Int. Ed. 53, 5821-5826 (2014).

36. Laughlin, S. T. \& Bertozzi, C. R. Metabolic labeling of glycans with azido sugars and subsequent glycan-profiling and visualization via Staudinger ligation. Nat. Protocols 2, 2930-2944 (2007).

37. Rudd, A. K., Valls Cuevas, J. M. \& Devaraj, N. K. SNAP-tag-reactive lipid anchors enable targeted and spatiotemporally controlled localization of proteins to phospholipid membranes. J. Am. Chem. Soc. 137, 4884-4887 (2015).

38. Nalluri, S. K. M. \& Ravoo, B. J. Light-responsive molecular recognition and adhesion of vesicles. Angew. Chem. Int. Ed. 49, 5371-5374 (2010).

39. Gong, Y.-H. et al. Photoresponsive smart template for reversible cell micropatterning. J. Mater. Chem. B 1, 2013-2017 (2013).

\section{Acknowledgements}

This work was supported by 973 Project (2012CB720602), and NSFC (21210002, 21431007, 21533008).

\section{Author contributions}

J.R. and X.Q. designed the research; P.S., J.E., Z.Y., N.G. and J.W. performed the research; P.S., J.E., Z.Y., N.G., J.W., Z.H. and Y.Z. analysed the data; P.S., J.R. and X.Q. wrote the paper.

\section{Additional information}

Supplementary Information accompanies this paper at http://www.nature.com/ naturecommunications

Competing financial interests: The authors declare no competing financial interests.

Reprints and permission information is available online at http://npg.nature.com/ reprintsandpermissions/

How to cite this article: Shi, P. et al. Spatiotemporal control of cell-cell reversible interactions using molecular engineering. Nat. Commun. 7, 13088 doi: $10.1038 /$ ncomms13088 (2016).

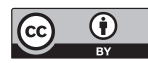

This work is licensed under a Creative Commons Attribution 4.0 International License. The images or other third party material in this article are included in the article's Creative Commons license, unless indicated otherwise in the credit line; if the material is not included under the Creative Commons license, users will need to obtain permission from the license holder to reproduce the material. To view a copy of this license, visit http://creativecommons.org/licenses/by/4.0/

(C) The Author(s) 2016 